\title{
Relevance of BCAR4 in tamoxifen resistance and tumour aggressiveness of human breast cancer
}

\author{
MFE Godinho', AM Sieuwerts ${ }^{2,3}$, MP Look ${ }^{2,3}$, D Meijer', JA Foekens ${ }^{2,3}$, LCJ Dorssers' and T van Agthoven*,I \\ 'Department of Pathology, Josephine Nefkens Institute, Erasmus MC-University Medical Center Rotterdam, Rotterdam, 3000 CA, The Netherlands; \\ ${ }^{2}$ Department of Medical Oncology, Josephine Nefkens Institute, Erasmus MC-University Medical Center Rotterdam, Rotterdam, 3000 CA, The \\ Netherlands; ${ }^{3}$ Cancer Genomics Center, Josephine Nefkens Institute, Erasmus MC-University Medical Center Rotterdam, Rotterdam, 3000 CA, \\ The Netherlands
}

BACKGROUND: Breast cancer anti-oestrogen resistance 4 (BCAR4) was identified in a search for genes involved in anti-oestrogen resistance in breast cancer. We explored whether BCAR4 is predictive for tamoxifen resistance and prognostic for tumour aggressiveness, and studied its function.

METHODS: BCAR4 mRNA levels were measured in primary breast tumours, and evaluated for association with progression-free survival (PFS) and clinical benefit in patients with oestrogen receptor (ER $\alpha$ )-positive tumours receiving tamoxifen as first-line monotherapy for advanced disease. In a separate cohort of patients with lymph node-negative, ER $\alpha$-positive cancer, and not receiving systemic adjuvant therapy, BCAR4 levels were evaluated for association with distant metastasis-free survival (MFS). The function of BCAR4 was studied with immunoblotting and RNA interference in a cell model.

RESULTS: Multivariate analyses established high BCAR4 mRNA levels as an independent predictive factor for poor PFS after start of tamoxifen therapy for recurrent disease. High BCAR4 mRNA levels were associated with poor MFS and overall survival, reflecting tumour aggressiveness. In BCAR4-expressing cells, phosphorylation of v-erb-b2 erythroblastic leukaemia viral oncogene homolog (ERBB)2, ERBB3, and their downstream mediators extracellular signal-regulated kinase I/2 and v-akt murine thymoma viral oncogene homolog (AKT) I/2, was increased. Selective knockdown of ERBB2 or ERBB3 inhibited proliferation, confirming their role in BCAR4induced tamoxifen resistance.

CONCLUSION: BCAR4 may have clinical relevance for tumour aggressiveness and tamoxifen resistance. Our cell model suggests that BCAR4-positive breast tumours are driven by ERBB2/ERBB3 signalling. Patients with such tumours may benefit from ERBB-targeted therapy.

British Journal of Cancer (2010) I 03, 1284-1291. doi:10.1038/sj.bjc.6605884 www.bjcancer.com

Published online 21 September 2010

(c) 2010 Cancer Research UK

Keywords: tamoxifen resistance; endocrine therapy; BCAR4; ERBB2; ERBB3; ERBB4

Over three decades, the anti-oestrogen tamoxifen has been the endocrine treatment of choice for patients with oestrogen receptor $(\mathrm{ER} \alpha)$-positive breast cancer (Early Breast Cancer Trialists' Collaborative Group, 1998). As an adjuvant therapy after surgery, tamoxifen reduces the incidence of relapse. In half of the patients with recurrent disease, tamoxifen induces an objective clinical response (Jaiyesimi et al, 1995; Jordan, 1995). However, the cancer will ultimately progress to hormone-independence that is, becoming unresponsive to tamoxifen. Despite extensive studies, the mechanisms involved in resistance are largely unknown (Dorssers et al, 1995; Clarke et al, 2003; Johnston et al, 2003; Nicholson et al, 2003; Osborne et al, 2005; Riggins et al, 2007).

Clinically, tamoxifen resistance is associated with poor prognosis and outcome. Thus, understanding of the mechanisms leading to this resistance is needed for developing new therapies. Previously, we applied several functional screens to identify genes involved in anti-oestrogen resistance (Dorssers et al, 1993;

*Correspondence: Dr T van Agthoven; E-mail: a.vanagthoven@erasmusmc.n Received 10 May 20 I0; revised 5 August 20 I0; accepted 6 August 20 I0; published online 2I September 2010
Van Agthoven et al, 1998; Brinkman et al, 2000; Meijer et al, 2006; Van Agthoven et al, 2009b). In one of the screens, we identified a new gene, breast cancer anti-oestrogen resistance 4 (BCAR4). In the tamoxifen-sensitive ZR-75-1 human breast cancer cell line, forced expression of BCAR4 induced tamoxifen-resistant proliferation (Meijer et al, 2006).

To establish the clinical relevance of $B C A R 4$, we investigated its relationship with tamoxifen resistance and cancer aggressiveness. In addition, we explored its biological function in vitro.

\section{MATERIALS AND METHODS}

\section{RNA isolation, complementary DNA synthesis and quantification of mRNA transcripts}

The isolation of RNA, quantification, complementary DNA synthesis, and normalisation to reference genes were performed as described before (Sieuwerts et al, 2005; details in the Supplementary Materials and Methods). TaqMan gene expression Assay-on-demand assays were BCAR4 Hs00415922_m1, epidermal 
growth factor receptor (EGFR; Hs01076091_m1), v-erb-b2 erythroblastic leukaemia viral oncogene homolog (ERBB)2 (Hs00170433_m1), ERBB3 (Hs00176538_m1) and ERBB4 (Hs00171783_m1) (Applied Biosystems International, Nieuwerkerk a/d Ijssel, the Netherlands), used according to the recommendations of the supplier.

\section{Cell lines}

The ZR-75-1 and derived cell lines containing expression vectors for BCAR4 (ZR/BCAR4; Meijer et al, 2006), BCAR3 (ZR/BCAR3; Van Agthoven et al, 1998), EGFR (ZR/EGFR; Van Agthoven et al, 1992), v-akt murine thymoma viral oncogene homolog (AKT)1 (ZR/AKT1) and AKT2 (ZR/AKT2; Van Agthoven et al, 2009b), were cultured as described previously (Van Agthoven et al, 1992).

\section{Western blot analysis and immunoprecipitation}

Immunoprecipitation and immunoblotting were performed as described previously (De Koning et al, 1996, details in the Supplementary Materials and Methods).

\section{Small interfering RNA-mediated inhibition of gene expression}

Cells were seeded into 96-well plates at a density of 7500 per well. After $24 \mathrm{~h}$, a mixture containing $25 \mu \mathrm{l}$ small interfering RNA (siRNA), $25 \mu \mathrm{l}$ DharmaFect3 (Perbio-Science, Etten Leur, the Netherlands), and $50 \mu \mathrm{l}$ medium was added. Final concentrations were $25 \mathrm{~nm}$ siRNA, $1 \mathrm{~nm} \beta$-estradiol or $1 \mu \mathrm{m} 4$-hydroxytamoxifen (Sigma-Aldrich Chemie, Zwijndrecht, the Netherlands). The ZR/EGFR cells were cultured in 4-hydroxyamoxifen-containing medium, with $10 \mathrm{ng} \mathrm{ml}^{1}$ EGF (Roche Diagnostics, Almere, the Netherlands). After 4 days, a WST-1 proliferation assay was performed (Roche Diagnostics). For each condition, six replicates were assayed. For RNA isolation, eight replicates were lysed with RNABee (Bio-Connect, Huissen, the Netherlands) and pooled. siRNAs were On TARGETplus SMARTpools (Dharmacon, Perbio-Science), each consisting of three oligonucleotides): EGFR (L-003114-00-0005), ERBB2 (L-003126-00-005), ERBB3 (L-00312700-0005) and ERBB4 (L003128-00-0005).

\section{Clinical details}

To assess the clinical relevance of BCAR4 in breast cancer, we measured mRNA levels in a cohort of 1474 ER $\alpha$-positive and -negative primary breast tumours from patients with detailed clinical follow-up (Van Agthoven et al, 2009a). BCAR4 was detected in 398 samples (27\%). ER $\alpha$ status was determined by ligand-binding or enzyme immunoassays (Foekens et al, 1989), $73 \%$ of the tumours were ER $\alpha$-positive. The medical ethics committee of the Erasmus MC-University Medical Center Rotterdam, the Netherlands, approved our study design (MEC 02.953). This retrospective study was in accordance with the Code of Conduct of the Federation of Medical Scientific Societies in the Netherlands, and is reported in line with the REMARK guidelines (McShane et al, 2006). All patients underwent surgery between 1979 and 1996.

To determine the association of BCAR4 with tamoxifen resistance, samples from 280 patients (selected from the cohort of 1474 patients) with $\mathrm{ER} \alpha$-positive tumours, who received firstline tamoxifen therapy for advanced disease, were analysed. About $42 \%$ of these patients had lymph node-negative cancer and $10 \%$ presented with metastasis at diagnosis. None had received adjuvant hormone therapy. A total of 53 patients were treated with systemic adjuvant chemotherapy (21 with anthracycline- and 32 with non-anthracycline-based regimens). Response to tamoxifen treatment was monitored according to a standardised protocol (Hayward et al, 1977; EORTC Breast Cancer Cooperative
Group, 2000). Clinical benefit, defined as objective (measurable) tumour response or no change for more than 6 months, was observed in 172 patients (62\%) with 11 complete and 37 partial remissions, and 124 had no change for more than 6 months. From the remaining 108 patients, 91 had progressive disease and 17 had no change for 6 months or less. The median follow-up time after the start of tamoxifen therapy was 38.2 months. The median time that $50 \%$ of the patients experienced progression is 9.2 months.

For studying the relation between BCAR4 mRNA levels and prognosis, 506 patients with lymph node-negative cancer, ER $\alpha$ protein-positive disease were selected from the cohort of 1474 patients. None received systemic adjuvant therapy. During follow-up, 193 experienced a relapse of distant metastasis (median follow-up time was 97 months). Patients with recurrent disease (115) were subsequently treated with tamoxifen. These were also included in the advanced study group of 280 patients.

\section{Statistical analyses}

Statistical computations were performed with STATA, 10.1 (STATA Corp., College Station, TX, USA). Differences in mRNA concentrations were assessed by the Mann-Whitney $U$ test or the Kruskal-Wallis test. Patient and tumour characteristics were used as grouping variables. Spearman rank correlation was used to quantify the strength of the monotonic association between continuous variables. For the levels of estrogen receptor (ESR)1 and progesterone receptor $(P G R)$, Box-Cox and logarithmic transformation was applied to reduce skewness. The transformed data were used for all analyses. The Cox proportional hazards model was used to calculate the hazard ratio (HR) and its $95 \%$ confidence interval in the analyses for metastasis-free survival (MFS), overall survival, progression-free survival (PFS) and postrelapse survival. For MFS, the end point was the first detection of a distant metastasis as confirmed after symptoms reported by the patient or at the time of detection of clinical signs at follow-up. This end point was preferred over relapse-free survival because relapse may be local and treated accordingly. The group is therefore more homogeneous from the perspective of treatment. During the years the tumours were collected (1979-1996), tumour grade was assessed by regional pathologists and not yet according current standards. In addition, approximately $30 \%$ of the pathology records lacked information on tumour grade. Therefore, we included in our prognostic analyses only the univariate survival data, because grade is included in the model for multivariate analysis. For all advanced patients treated with tamoxifen, PFS was defined as the time elapsed between initiation of first-line tamoxifen therapy and the first detection of disease progression. In multivariable analyses for PFS, the model included the classical predictive factors age, menopausal status at start of first-line therapy, the disease-free interval, the dominant site of relapse and ESR1 and PGR mRNA levels. Proportional hazards assumption was verified by a test based on Schoenfeld residuals. In case of violation, the analysis was stratified for the variable. Data were visualised in survival curves with the method of Kaplan and Meier. The logrank test was used to compare survival curves, whereas for more than two groups the logrank test for trend was used. Logistic regression analysis was used for the relation between mRNA levels and clinical benefit of tamoxifen therapy and reported as the odds ratio and its $95 \%$ confidence interval. A two-sided $P$-value of $<0.05$ was considered statistically significant.

\section{RESULTS}

\section{Clinical relevance of BCAR4}

Association of BCAR4 mRNA levels with tamoxifen resistance To address the question whether BCAR4 is associated with clinical 
tamoxifen resistance, we studied $280 \mathrm{ER} \alpha$-positive primary breast cancer specimens from patients with advanced disease. These patients received tamoxifen monotherapy as first-line treatment. The levels of BCAR4 mRNAs were determined by quantitative RT - PCR of complementary DNA preparations of primary breast tumours. The levels of BCAR4 mRNA were analysed for association with the clinicopathological factors and the end points PFS, clinical benefit and post-relapse survival. BCAR4 mRNA was detected in 81 samples (29\%). Tumours with mRNA levels below the detection limit were categorised as negative. Tumours with detectable levels of mRNA were categorised in a single group (positive), or in two groups (low or high) split at median levels. No relation between categorised BCAR4 mRNA levels and age, menopausal status, tumour size, nodal status, or adjuvant systemic treatment of the patients was observed (Supplementary Table 1).

Univariate Cox regression analysis revealed that the presence of BCAR4 mRNA was significantly associated with shorter PFS (positive vs negative $\mathrm{HR}=1.45, P=0.007$, Table 1 ). High levels were significantly associated with shorter PFS (high $v s$ negative $\mathrm{HR}=1.70$, $P=0.003$ ), whereas low levels were not informative. The KaplanMeier curves for PFS in these subgroups show rapid progression of the disease in patients with high levels of BCAR4 (Figure 1).

In the multivariate analysis for PFS, high BCAR4 levels were independently predictive for short PFS (high $v s$ negative, $\mathrm{HR}=1.47, P=0.041$, Table 1 ). In the univariate analysis of postrelapse survival, high BCAR4 levels were related with poor outcome (high $v s$ negative $\mathrm{HR}=1.68, P=0.007$, Supplementary Table 2), but this association was not independent of the traditional predictive factors $(\mathrm{HR}=1.44, P=0.073)$. In the univariate logistic regression analysis of clinical benefit, high $B C A R 4$ levels were significantly associated with an unfavourable response to tamoxifen treatment (high $v s$ negative odds ratio $=0.49, P=0.042$, Supplementary Table 3).
Association of BCAR4 $\mathrm{mRNA}$ levels with tumour aggressiveness In our cell line model, expression of BCAR4 gives rise to an aggressive phenotype, that is, vigorous oestrogen-independent growth and anchorage independence (Meijer et al, 2006). Therefore, we investigated whether BCAR4 mRNA levels give information on tumour aggressiveness. To estimate the true prognostic value of $B C A R 4$, we performed analyses on a cohort of 506 patients with lymph node-negative cancer, ER $\alpha$-positive disease. These patients

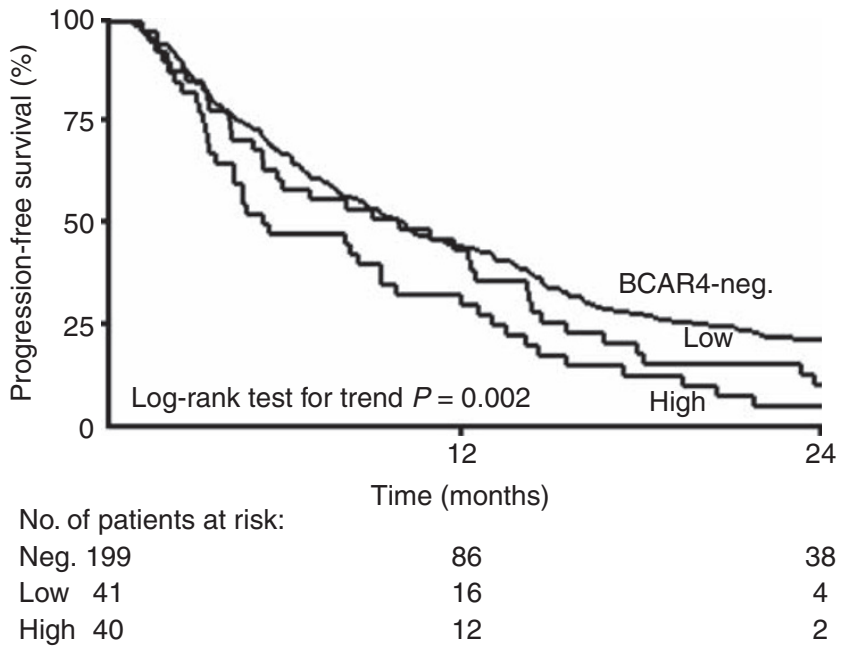

Figure I Progression-free survival of 280 patients with advanced disease treated with first-line tamoxifen monotherapy. Kaplan-Meier curves for PFS for subgroups of patients as a function of BCAR4 mRNA status. Patients at risk at 12-month intervals are indicated.

Table I PFS after first-line tamoxifen treatment of 280 patients with oestrogen receptor-positive primary breast cancer

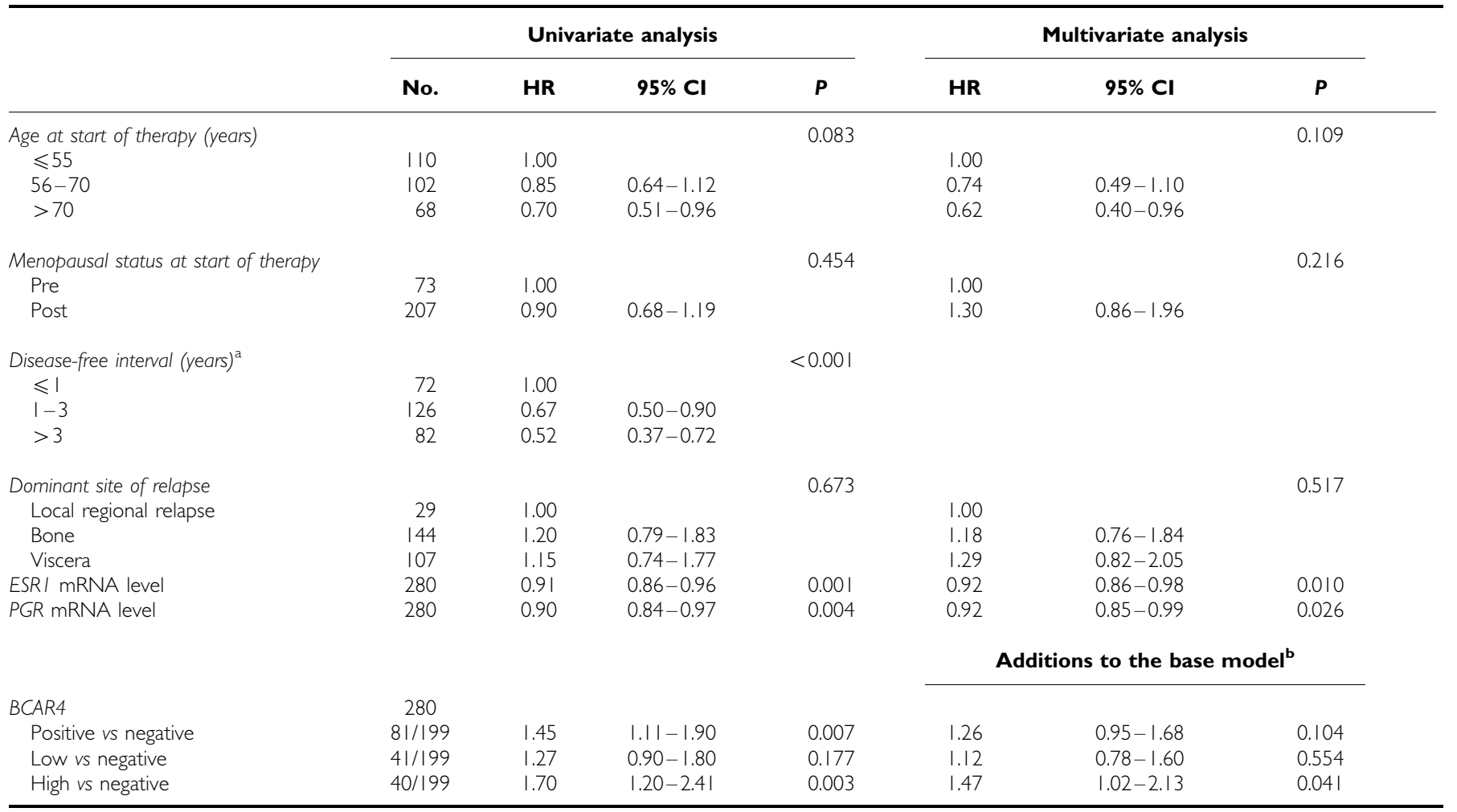

Abbreviations: $\mathrm{Cl}=$ confidence interval; $\mathrm{HR}=$ hazard ratio; PFS = progression-free survival. BCAR4 mRNA levels were defined as high, low or negative. ${ }^{a}$ Multivariate analyses were stratified for this variable. ${ }^{b} \mathrm{BCAR} 4$ was introduced to the base model that included the factors age, menopausal status, dominant site of relapse and ESRI and PGR mRNA levels as transformed continuous variables. Inclusion of adjuvant chemotherapy in the base model did not change the estimates for BCAR4. 
had not received systemic adjuvant treatment. BCAR4 mRNA was detected in 119 samples (24\%). No relation between categorised BCAR4 mRNA levels and age, menopausal status, tumour size, or tumour grade was found (Supplementary Table 4).

Univariate analyses showed a significant association between the presence of BCAR4 mRNA (positive vs negative) and shorter MFS $(\mathrm{HR}=1.41, P=0.033)$ and overall survival $(\mathrm{HR}=1.77, P=0.001$; Table 2). Kaplan-Meier curves show the rapid recurrence of the disease in the group of patients with detectable BCAR4 compared with the BCAR4-negative group (Figure 2). Patients with high BCAR4 in the tumours showed the worst MFS and overall survival (Table 2).

\section{Characterisation of BCAR4 using a breast cancer model}

BCAR4 activates ERBB2 and ERBB3 signalling Identification of activated proteins in BCAR4-expressing cells may give important insight into BCAR4 function in anti-oestrogen-resistant proliferation. To identify proteins activated by BCAR4, we performed immunoprecipitation using an antibody directed to phosphorylated tyrosine residues. Lysates of ZR-75-1 cells were compared with lysates of equal numbers of cells with forced expression of BCAR4, BCAR3, AKT1 or AKT2. All these transgenic cell lines are resistant to tamoxifen, due to expression of the transgene (Van Agthoven et al, 1998; Brinkman et al, 2000; Meijer et al, 2006; Van Agthoven et al, 2009b). Among others, an abundant $180-\mathrm{kD}$ band was detected in ZR/BCAR4 cells, which was hardly observed in the other cell lines (Figure 3A, arrow). The position of the protein band and the knowledge that the ERBB receptors can be involved in tamoxifen resistance provided a possible clue. Identically loaded blots were probed with antibodies against EGFR, ERBB2, ERBB3 and ERBB4. ZR-75-1 cells do not express EGFR (Van Agthoven et al, 1992) and phosphorylated EGFR was not detected. Low levels of phosphorylated ERBB4 were detected, but no increase in ZR/BCAR4 cells (Figure 3A). In contrast, phosphorylation of
ERBB2 and ERBB3 was noticeably elevated in the ZR/BCAR4 cells (Figure 3A). This increase in phosphorylation of ERBB2 and ERBB3 in BCAR4 transduced cells, compared with empty vectorexpressing cells, was not due to higher total levels of these receptor proteins (Figure 3B). Additional analyses showed that AKT and extracellular signal-regulated kinase $1 / 2$, representing major proliferative and survival pathways downstream of ERBB2/ERBB3 signalling, were also activated in ZR/BCAR4 cells (Figure 3B).

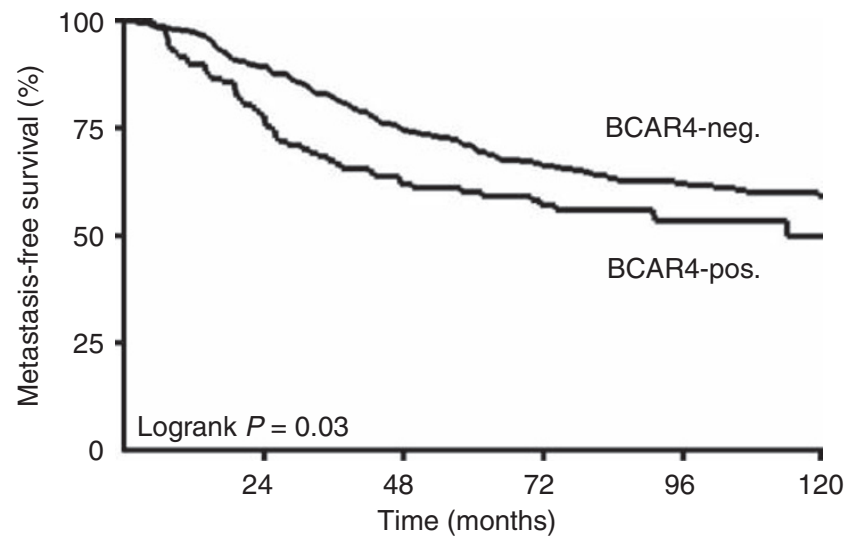

No. of patients at risk:

$\begin{array}{lcccccc}\text { Neg. } & 387 & 332 & 258 & 192 & 134 & 69 \\ \text { Pos. } & 119 & 90 & 68 & 53 & 34 & 11\end{array}$

Figure 2 Metastasis-free survival in 506 patients with $L N N$, ER $\alpha$-positive breast cancer. Kaplan-Meier curves for MFS for subgroups of patients as a function of BCAR4 mRNA status of the primary tumours. Patients at risk at 24-month intervals are indicated.

Table 2 Univariate analysis for metastasis-free and overall survival in 506 patients with oestrogen receptor-positive, lymph node-negative primary breast cancer

\begin{tabular}{|c|c|c|c|c|c|c|c|}
\hline & \multicolumn{4}{|c|}{ Metastasis-free survival } & \multicolumn{3}{|c|}{ Overall survival } \\
\hline $\begin{array}{l}\text { Tumour size }(\mathrm{cm}) \\
\quad \leqslant 2 \\
>2\end{array}$ & $\begin{array}{l}233 \\
273\end{array}$ & $\begin{array}{l}1.00 \\
1.17\end{array}$ & $0.88-1.56$ & 0.269 & $\begin{array}{l}1.00 \\
1.20\end{array}$ & $0.89-1.62$ & 0.232 \\
\hline $\begin{array}{l}\text { BCAR4 } \\
\text { Positive vs negative } \\
\text { Low vs negative } \\
\text { High vs negative }\end{array}$ & $\begin{array}{r}119 / 387 \\
60 / 387 \\
59 / 387\end{array}$ & $\begin{array}{l}1.41 \\
1.29 \\
1.54\end{array}$ & $\begin{array}{l}1.03-1.94 \\
0.84-1.98 \\
1.03-2.31\end{array}$ & $\begin{array}{l}0.033 \\
0.240 \\
0.037\end{array}$ & $\begin{array}{l}1.77 \\
1.62 \\
1.94\end{array}$ & $\begin{array}{l}1.28-2.45 \\
1.06-2.48 \\
1.28-2.94\end{array}$ & $\begin{array}{l}0.001 \\
0.027 \\
0.002\end{array}$ \\
\hline
\end{tabular}

Abbreviations: $\mathrm{Cl}=$ confidence interval; $\mathrm{HR}=$ hazard ratio; MFS = metastasis-free survival. BCAR4 mRNA levels were defined as high, low or negative. 
A

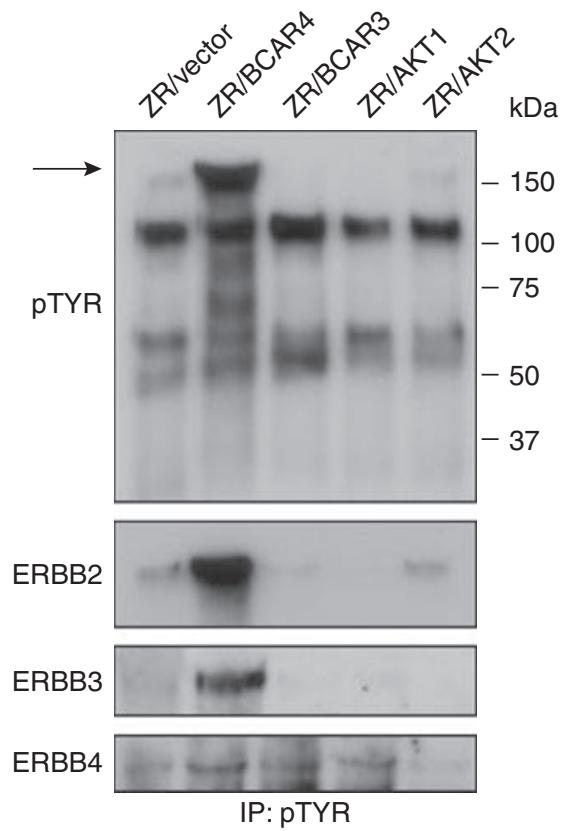

B

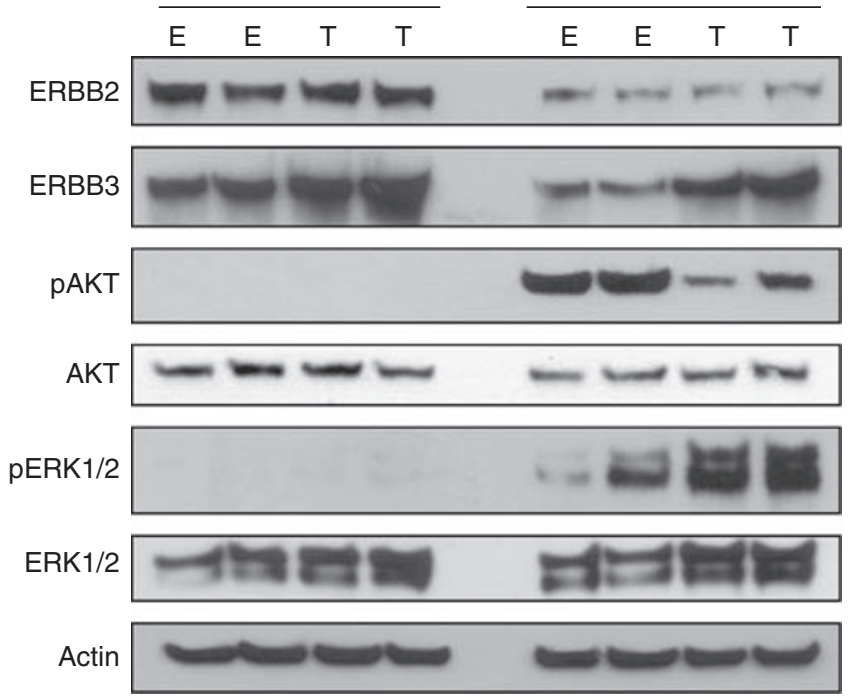

Figure 3 Activation of the ERBB2/ERBB3 signalling pathway by BCAR4 (A) Lysates of ZR-75-I cells transduced with empty vector, BCAR4, BCAR3, $A K T 1$ or AKT2 were immunoprecipitated with phosphotyrosine-specific antibody and subjected to western blot analysis. An approximately 180-kD band in lysates from ZR/BCAR4 cells is marked with an arrow. To identify the phosphorylated proteins in this band, identically loaded blots were probed with antibodies against EGFR, ERBB2, ERBB3 and ERBB4. PhosphoEGFR was not detected (data not shown). (B) Activation of downstream signalling of ERBB2/ERBB3. Lysates of two independent pools of ZR-75- I cells containing empty vector or stably expressing BCAR4, cultured in the presence of oestradiol (E) or 4-OHT (T), were subjected to western blot analysis. Blots were probed with total ERBB2, ERBB3, phospho-AKT, totalAKT, phospho-extracellular signal-regulated kinase (ERK)I/2 and totalERKI/2 antibodies and $\beta$-actin for loading control.

This indicates that BCAR4 expression enhances the activity of the $\mathrm{ERBB} 2$ and ERBB3 receptors.

siRNA-mediated reduction of ERBB signalling inhibits BCAR4induced proliferation If increased ERBB2 and/or ERBB3 signalling is required for BCAR4-induced tamoxifen resistance then inhibition of ERBB2 and/or ERBB3 should suppress ZR/BCAR4 proliferation. To test this, ZR/BCAR4 and control cell lines were transfected with small interfering RNAs (siRNA) directed against the different ERBB receptors. Successful downregulation of ERBB2 was confirmed by western blot analysis (insert, Figure 4A). Inhibition of all ERBB receptors was verified by quantitative RT - PCR, showing reductions of over $50 \%$ of EGFR in ZR/EGFR cells, $\geqslant 80 \%$ of $E R B B 2$ and $E R B B 3$, and more than $60 \%$ for $E R B B 4$ mRNA transcripts.

The ZR-75-1 cells are oestrogen-dependent, and growth is inhibited by anti-oestrogens. As a consequence, addition of 4-hydroxytamoxifen inhibited proliferation of ZR-75-1 cells, irrespective of the addition of siRNA (Figure 4A). Knockdown of EGFR expression did not inhibit proliferation of ZR/BCAR4 cells, due to the fact that both our parental and ZR/BCAR4 cells are devoid of EGFR. However, ERBB2, ERBB3, and ERBB4 siRNA all significantly inhibited proliferation of ZR/BCAR4 cells in the presence of 4-hydroxytamoxifen (Figure 4A). In the presence of EGF, ZR/EGFR cells are resistant to tamoxifen due to the expression of the EGFR transgene (Van Agthoven et al, 1992). Proliferation was reduced when EGFR was inhibited by siRNAmediated interference (Figure 4A) confirming the involvement of this pathway in resistance of these cells. The knockdown of ERBB2 and ERBB4, but not ERBB3, in ZR/EGFR cells also resulted in the inhibition of proliferation.

In oestradiol-containing medium, proliferation of BCAR4expressing cells was also inhibited by addition of $E R B B 2$ or ERBB3 siRNA (Figure 4B). In contrast, knocking down ERBB2 or ERBB3 expression in the vector-control cells did not inhibit proliferation. In the presence of oestrogen, ZR/EGFR cells use the oestrogen receptor pathway and are not further stimulated with EGF. As expected, knockdown of EGFR in the presence of oestradiol did not inhibit proliferation (Figure 4B). In oestradiol-containing medium, proliferation of ZR-75-1, ZR/BCAR4 and ZR/EGFR cells was reduced by knockdown of ERBB4, suggesting a role of ERBB4 in oestrogen-regulated growth (Figure $4 \mathrm{~B}$ ).

\section{DISCUSSION}

We describe two sets of patients with ER $\alpha$-positive breast cancer. The first group received tamoxifen as first-line treatment for advanced disease. This allowed for the analysis of clinical benefit, PFS and post-relapse survival in advanced breast cancer patients in relation to BCAR4 mRNA levels in the primary tumour. In the second group, including only patients with lymph node-negative cancer, $\mathrm{ER} \alpha$-positive breast cancer, we investigated the prognostic value of BCAR4 mRNA levels. Our data show that high BCAR4 levels are independently predictive for shorter PFS after start of first-line tamoxifen therapy and in addition provide prognostic information for MFS and overall survival. Our results indicate that patients with high levels of BCAR4 are at increased risk for early recurrence of the disease and have reduced probability of longterm benefit of tamoxifen treatment.

We show that ERBB2/ERBB3 signalling is critically involved in the mechanism of BCAR4-induced proliferation in the presence of oestrogen or tamoxifen. The overexpression of BCAR4 induced strong phosphorylation of ERBB2 and ERBB3. Key downstream mediators of ERBB signalling, AKT and extracellular signalregulated kinase $1 / 2$, were also activated in $B C A R 4$-transduced cells. The siRNA-mediated inhibition of the ERBB receptors confirmed the direct involvement of ERBB2 and ERBB3 in BCAR4-mediated proliferation. The ERBB tyrosine kinase receptors have important roles in normal development, growth, and differentiation. Their involvement in numerous types of human tumours has been reported (for review, see Holbro et al, 2003). Gene amplification and overexpression of ERBB2 has been reported in several types of cancer, including the breast cancer, and has been shown to contribute to a poor clinical outcome 
A

$\mathrm{OH}-\mathrm{TAM}$

$\mathrm{OH}-\mathrm{TAM}+\mathrm{EGF}$

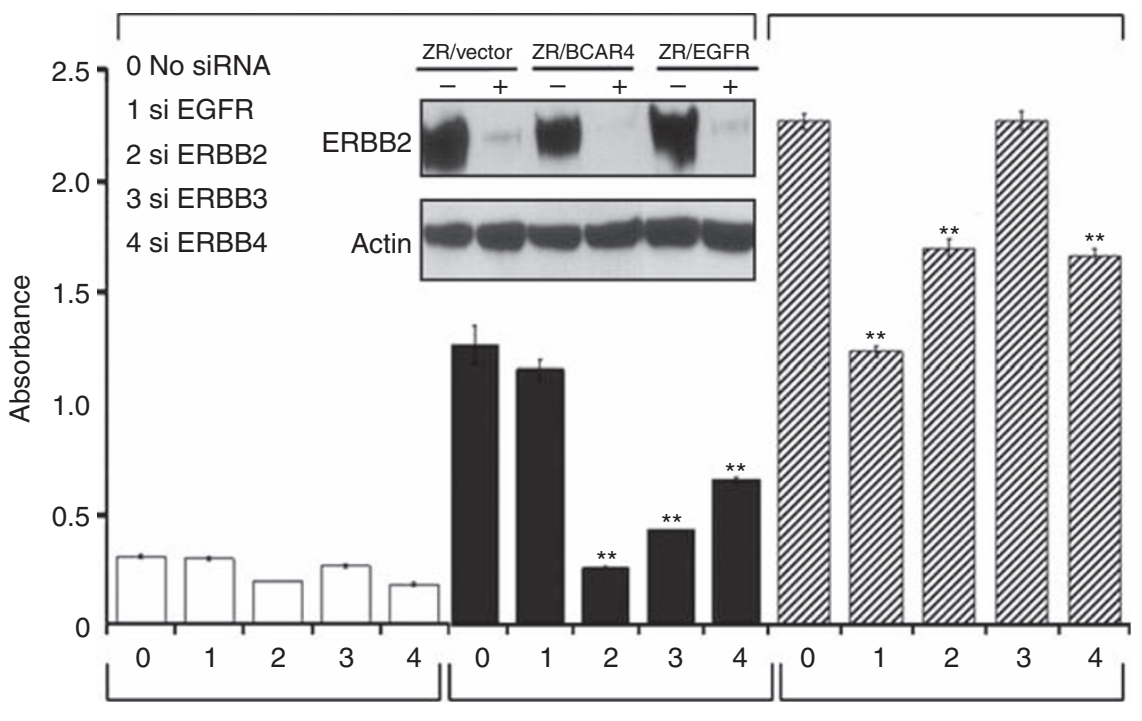

ZR/vector

ZR/BCAR4

ZR/EGFR

B

E2

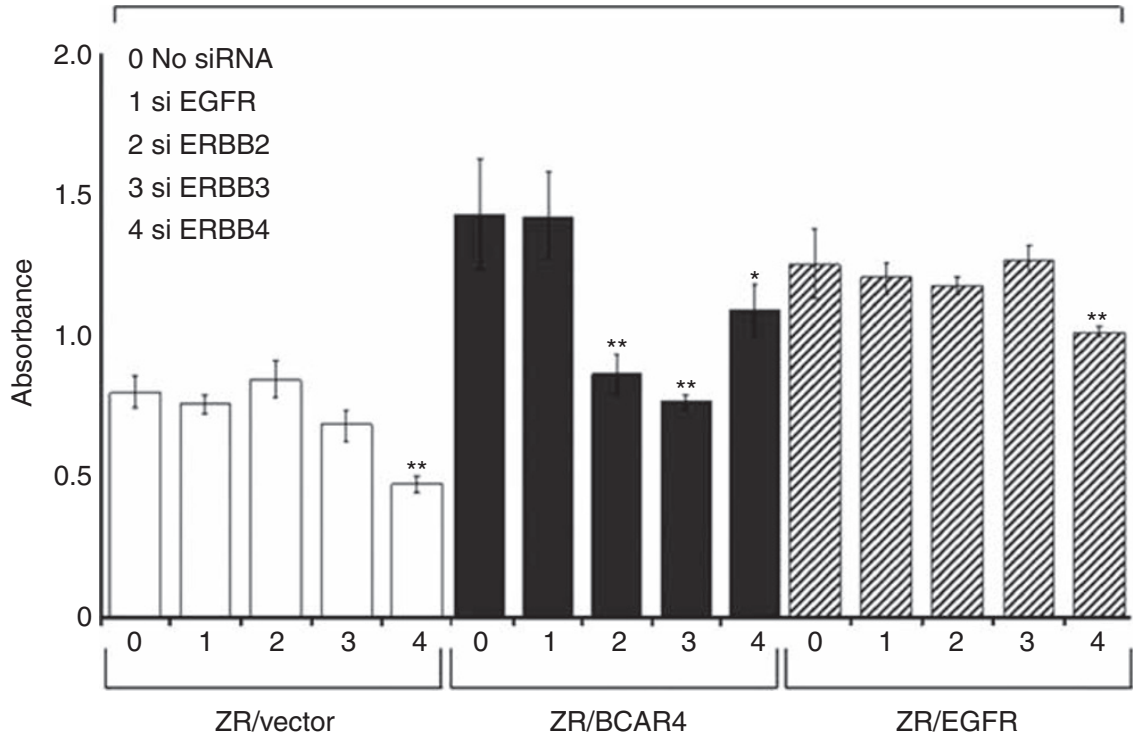

Figure 4 Knockdown of ERBB receptors reduces proliferation of cells with forced expression of BCAR4 or EGFR. (A) Cells were exposed to 4-hydroxy tamoxifen (OH-TAM), plus EGF for ZR/EGFR cells. Insert shows the down-regulation of ERBB2 protein by western blot analysis, $96 \mathrm{~h}$ after treatment with siERBB2 $(+)$ or transfection reagent only $(-)$. (B) Proliferation was measured in oestradiol (E2)-containing medium. Bars represent the average of 6 independent siRNA transfections. Data of six replicates is reported as mean \pm s.d.. Significance was determined by the Mann-Whitney $U$ test; $* P<0.05$, *** $P<0.0$ I compared to the control (medium without siRNA).

(Slamon et al, 1989; Gullick et al, 1991; Seshadri et al, 1993; Berns et al, 1995; Liu et al, 2007; Van Agthoven et al, 2009a). Overexpression or amplification of ERBB2 predicts response failure to tamoxifen therapy (Wright et al, 1992; Berns et al, 1995; Dowsett et al, 2001; Hurtado et al, 2008; Van Agthoven et al, 2009a). In patients with hormone receptor-positive breast cancer who had received adjuvant tamoxifen therapy, EGFR, ERBB2 and ERBB3 phosphorylation was observed to be associated with shorter disease-free and overall survival (Frogne et al, 2009).

We have shown a mechanistic relationship between BCAR4 and the ERBB2/ERBB3 signalling pathways in the development of anti-oestrogen resistance in vitro. Therefore, BCAR4 expression may identify a subgroup of patients with increased ERBB2/ ERBB3 signalling, independent of gene amplification and/or over expression of ERBB2. This is particularly important because currently the application of ERBB2-targeting drugs is limited to patients whose tumours express high levels of the ERBB2 protein or show amplification of the ERBB2 gene (Hynes and Lane, 2005). Tumours with activated ERBB2 signalling via other mechanisms than amplification or over expression will be tested ERBB2negative. These patients are currently withheld ERBB2-targeting drugs. However, it remains to be established whether BCAR4positive primary tumours have elevated levels of phosphorylated ERBB2 or ERBB3.

The BCAR4 amino-acid sequence predicts two transmembrane domains, suggesting it may be localised at the cell membrane. Therefore, it might be a ligand for ERBB3, stimulating ERBB2/ ERBB3 activity. It could also be a substrate for membrane-bound 
members of the ADAM (a disintegrin and metalloproteinase) domain family of proteins, some of them reported to be expressed in several cancers, including the breast cancer, in which they may release ERBB ligands and promote proliferation (for review, see Mochizuki and Okada, 2007). Another possible mechanism could be an intracellular interaction with the different ERBB receptors inducing their phosphorylation, as reported for nucleolin (Di Segni et al, 2008). The BCAR4 protein could also function similarly to Mucin4 that was reported to be a transmembrane ligand for ERBB2 (for review, see Carraway et al, 2001). Another ability of Mucin4 is to translocate ERBB2 to the apical surface in polarised epithelial cells (Ramsauer et al, 2003), or to increase the amount of ERBB2 and ERBB3 in the plasma membrane, by preventing their intracellular accumulation (Funes et al, 2006). How BCAR4 activates ERBB2/ERBB3 signalling remains to be established.

In conclusion, high levels of $B C A R 4$ mRNA predict resistance to endocrine therapy and poor outcome in ER $\alpha$-positive breast cancer. If $B C A R 4$-positive tumours are driven by ERBB2 signalling, in the absence of gene amplification or overexpression, as our results suggest, then more patients may benefit from ERBB2directed therapy.

\section{ACKNOWLEDGEMENTS}

We thank Marion Meijer-van Gelder for the evaluation of the clinical data. This research was supported by the Dutch Cancer Society, the Association for International Cancer Research (04-0148), Erasmus MC Grants 1998 and 2007 and The Netherlands Genomics Initiative/The Netherlands Organization for Scientific Research.

\section{Conflict of interest}

The authors declare no conflict of interest.

Supplementary Information accompanies the paper on British Journal of Cancer website (http://www.nature.com/bjc)

\section{REFERENCES}

Berns EMJJ, Foekens JA, Van Staveren IL, Van Putten WLJ, De Koning HYWCM, Portengen H, Klijn JGM (1995) Oncogene amplification and prognosis in breast cancer: relationship with systemic treatment. Gene 159: $11-18$

Brinkman A, Van der Flier S, Kok EM, Dorssers LCJ (2000) BCAR1, a human homologue of the adapter protein p130Cas and antiestrogen resistance in breast cancer cells. J Natl Cancer Inst 92: 112-120

Carraway KL, Price-Schiavi SA, Komatsu M, Jepson S, Perez A, Carraway CA (2001) Muc4/sialomucin complex in the mammary gland and breast cancer. J Mammary Gland Biol Neoplasia 6: 323-337

Clarke R, Liu MC, Bouker KB, Gu Z, Lee RY, Zhu Y, Skaar TC, Gomez B, O'Brien K, Wang Y, Hilakivi-Clarke LA (2003) Antiestrogen resistance in breast cancer and the role of estrogen receptor signaling. Oncogene 22: $7316-7339$

De Koning JP, Schelen AM, Dong F, Van Buitenen C, Burgering BMT, Bos JL, Löwenberg B, Touw IP (1996) Specific involvement of tyrosine 764 of human granulocyte colony-stimulating factor receptor in signal transduction mediated by p145/Shc/GRB2 or p90/GRB2 complexes. Blood 87: $132-140$

Di Segni A, Farin K, Pinkas-Kramarski R (2008) Identification of nucleolin as new ErbB receptors-interacting protein. PLoS ONE 3: e2310

Dorssers LCJ, Van Agthoven T, Brinkman A (1995) Involvement of genetic alterations in breast tumour progression to hormone independence in vitro. Endocrine Related Cancer 2: 123-126

Dorssers LCJ, Van Agthoven T, Dekker A, Van Agthoven TLA, Kok EM (1993) Induction of antiestrogen resistance in human breast cancer cells by random insertional mutagenesis using defective retroviruses: Identification of bcar-1, a common integration site. Mol Endocrinol 7: $870-878$

Dowsett M, Harper-Wynne C, Boeddinghaus I, Salter J, Hills M, Dixon M, Ebbs S, Gui G, Sacks N, Smith I (2001) HER-2 amplification impedes the antiproliferative effects of hormone therapy in estrogen receptor-positive primary breast cancer. Cancer Res 61: $8452-8458$

Early Breast Cancer Trialists' Collaborative Group (1998) Tamoxifen for early breast cancer: an overview of the randomised trials. Lancet 351: $1451-1467$

EORTC Breast Cancer Cooperative Group (2000) Manual for Clinical Research and Treatment in Breast Cancer pp. 116-117. Excerpta Medical: Almere, The Netherlands

Foekens JA, Portengen H, van Putten WL, Peters HA, Krijnen HL, AlexievaFigusch J, Klijn JG (1989) Prognostic value of estrogen and progesterone receptors measured by enzyme immunoassays in human breast tumour cytosols. Cancer Res 49: 5823-5828

Frogne T, Laenkholm AV, Lyng MB, Henriksen KL, Lykkesfeldt AE (2009) Determination of HER2 phosphorylation at tyrosine 1221/1222 improves prediction of poor survival for breast cancer patients with hormone receptor-positive tumours. Breast Cancer Res 11: R11
Funes M, Miller JK, Lai C, Carraway III KL, Sweeney C (2006) The mucin Muc4 potentiates neuregulin signaling by increasing the cell-surface populations of ErbB2 and ErbB3. J Biol Chem 281: 19310-19319

Gullick WJ, Love SB, Wright C, Barnes DM, Gusterson B, Harris AL, Altman DG (1991) c-erbB-2 protein overexpression in breast cancer is a risk factor in patients with involved and uninvolved lymph nodes. Br J Cancer 63: $434-438$

Hayward JL, Carbone PP, Heuson JC, Kumaoka S, Segaloff A, Rubens RD (1977) Assessment of response to therapy in advanced breast cancer: a project of the Programme on Clinical Oncology of the International Union Against Cancer, Geneva, Switzerland. Cancer 39: 1289-1293

Holbro T, Civenni G, Hynes NE (2003) The ErbB receptors and their role in cancer progression. Exp Cell Res 284: 99-110

Hurtado A, Holmes KA, Geistlinger TR, Hutcheson IR, Nicholson RI, Brown M, Jiang J, Howat WJ, Ali S, Carroll JS (2008) Regulation of ERBB2 by oestrogen receptor-PAX2 determines response to tamoxifen. Nature 456: $663-666$

Hynes NE, Lane HA (2005) ERBB receptors and cancer: the complexity of targeted inhibitors. Nat Rev Cancer 5: $341-354$

Jaiyesimi IA, Buzdar AU, Decker DA, Hortobagyi GN (1995) Use of tamoxifen for breast cancer: Twenty-eight years later. J Clin Oncol 13: 513-529

Johnston SR, Head J, Pancholi S, Detre S, Martin LA, Smith IE, Dowsett M (2003) Integration of signal transduction inhibitors with endocrine therapy: an approach to overcoming hormone resistance in breast cancer. Clin Cancer Res 9: 524S-532S

Jordan VC (1995) Third annual William L McGuire memorial lecture - Studies on the estrogen receptor in breast cancer - 20 years as a target for the treatment and prevention of cancer. Breast Cancer Res Treat 36: $267-285$

Liu B, Ordonez-Ercan D, Fan Z, Edgerton SM, Yang X, Thor AD (2007) Downregulation of erbB3 abrogates erbB2-mediated tamoxifen resistance in breast cancer cells. Int J Cancer 120: $1874-1882$

McShane LM, Altman DG, Sauerbrei W, Taube SE, Gion M, Clark GM, Statistics Subcommittee of N.C.I.E.W.G.o.C.D (2006) Reporting recommendations for tumour MARKer prognostic studies (REMARK). Breast Cancer Res Treat 100: 229-235

Meijer D, Van Agthoven T, Bosma PT, Nooter K, Dorssers LCJ (2006) Functional screen for genes responsible for tamoxifen resistance in human breast cancer cells. Mol Cancer Res 4: 379-386

Mochizuki S, Okada Y (2007) ADAMs in cancer proliferation and progression. Cancer Sci 95: 621-628

Nicholson RI, Gee JM, Knowlden J, McClelland R, Madden TA, Barrow D, Hutcheson I (2003) The biology of antihormone failure in breast cancer. Breast Cancer Res Treat 80(Suppl 1): S29-S34; discussion S35

Osborne CK, Shou J, Massarweh S, Schiff R (2005) Crosstalk between estrogen receptor and growth factor receptor pathways as a cause for endocrine therapy resistance in breast cancer. Clin Cancer Res 11: $865 s-8670$ s 
Ramsauer VP, Carraway CA, Salas PJ, KL C (2003) Muc4/sialomucin complex, the intramembrane ErbB2 ligand, translocates ErbB2 to the apical surface in polarized epithelial cells. J Biol Chem 278: $30142-30147$

Riggins RB, Schrecengost RS, Guerrero MS, Bouton AH (2007) Pathways to tamoxifen resistance. Cancer Lett 256: 1-24

Seshadri R, Firgaira FA, Horsfall DJ, McCaul K, Setlur V, Kitchen P (1993) Clinical significance of HER-2/neu oncogene amplification in primary breast cancer. J Clin Oncol 11: 1936-1942

Sieuwerts AM, Meijer-van Gelder ME, Timmermans M, Trapman AM, Garcia RR, Arnold M, Goedheer AJ, Portengen H, Klijn JG, Foekens JA (2005) How ADAM-9 and ADAM-11 differentially from estrogen receptor predict response to tamoxifen treatment in patients with recurrent breast cancer: a retrospective study. Clin Cancer Res 11: 7311-7321

Slamon DJ, Clark GM, Wong SG, Levin WJ, Ullrich A, McGuire WL (1989) Human breast cancer: correlation of relapse and survival with amplification of HER2/neu oncogene. Science 235: 177-182

Van Agthoven T, Sieuwerts AM, Meijer-Van Gelder ME, Look MP, Smid M, Veldscholte J, Sleijfer S, Foekens JA, Dorssers LCJ (2009a) Relevance of breast cancer antiestrogen resistance genes in human breast cancer progression and tamoxifen resistance. J Clin Oncol 27: 542-549

Van Agthoven T, Van Agthoven TLA, Dekker A, Van der Spek PJ, Vreede L, Dorssers LCJ (1998) Identification of BCAR3 by a random search for genes involved in antiestrogen resistance of human breast cancer cells. EMBO J 17: 2799-2808

Van Agthoven T, Van Agthoven TLA, Portengen H, Foekens JA, Dorssers LCJ (1992) Ectopic expression of epidermal growth factor receptors induces hormone independence in ZR-75-1 human breast cancer cells. Cancer Res 52: $5082-5088$

Van Agthoven T, Veldscholte J, Smid M, Van Agthoven TLA, Vreede L, Broertjes M, De Vries I, De Jong D, Sarwari R, Dorssers LCJ (2009b) Functional identification of genes causing estrogen independence of human breast cancer cells. Breast Cancer Res Treat 114: 23-30

Wright C, Nicholson S, Angus B, Sainsbury JRC, Farndon J, Cairns J, Harris AL, Horne CHW (1992) Relationship between c-erbB-2 protein product expression and response to endocrine therapy in advanced breast cancer. $\mathrm{Br}$ J Cancer 65: $118-121$ 